\title{
STUDY ON PLASMA D-DIMER FOR EARLY DIAGNOSIS OF ISCHEMIC STROKE
}

\author{
SARKAR PK ${ }^{1}$, SAHA K ${ }^{2}$, BAQUI M ${ }^{1}$, CHOWDHURY MSJH ${ }^{1}$, ALAMGIR MA ${ }^{3}$, IQBAL MR ${ }^{4}$, GHOSE \\ $\mathrm{SK}^{5}$, HABIB $\mathrm{M}^{6}$, HOQUE MA ${ }^{7}$, MONDAL BA ${ }^{7}$, QURAISHI FA ${ }^{7}$, MOHAMMAD QD $^{8}$
}

\begin{abstract}
Background: Plasma D-Dimer is a biomarker of thrombo-embolism and a sensitive indicator of subclinical coagulopathy. Its level begins to rise within the first 6 hours of onset of symptoms of acute ischemic stroke. The availability of a rapid diagnostic test like plasma D-Dimer in acute ischemic stroke would be a substantial adjunct to CT and MRI. The test for plasma D-Dimer is quick and easy to perform based on principle of latex agglutination. This study is taken to estimate Plasma D-Dimer level in ischemic stroke patients and to see its role in early diagnosis, and also to differentiate different types of acute ischemic stroke in Bangladeshi population.

Methods: This case control study was conducted in Dhaka Medical College Hospital (DMCH) from July 2010 to June 2012. Plasma D-Dimer was estimated by latex agglutination method in $D M C H$ laboratory. Fifty stroke patients (age, 58.36 14.8 years; sex-M/F: 34/16) and 50 age matched control (58.80 15.01 years; sex-M/F: 36/14) were enrolled. In ischemic stroke group all patients were presented from 4 hours to 3 days. CT scan of brain revealed both cortical and subcortical lesion (56\%), subcortical lesion (34\%) and cortical lesion (10\%). The CT volume of the involved brain lesion was $19.95 \pm 4.92 \mathrm{~cm}^{3}$. The value of plasma D-Dimer were evaluated in both control and ischemic stroke patients, correlated with CT size of infract and different types of acute ischemic strokes.

Results: Qualitative analysis of plasma D-Dimer reveals $76 \%$ positive and $24 \%$ negative in the ischemic stroke group. On the other hand, plasma D-Dimer value was only 6\% positive and 94\% negative in the control group. Qualitative analysis of plasma D-Dimer differs significantly $\left(\chi^{2}=50.64\right.$; $p=0.001$ ) between the two groups. The analysis also reveals $76 \%$ sensitivity and $94 \%$ specificity of the Plasma D-Dimer test. Quantitative measurement of plasma D-Dimer ( $p=0.001$ ) was $804 \pm 120$ $\mathrm{\eta g} / \mathrm{ml}$ and $126 \pm 16 \mathrm{ng} / \mathrm{ml}$ in ischemic and control groups respectively. The plasma concentration of D-Dimer between the two groups was statistically highly significant ( $t=31.21 ; p=0.001$ ). Mean plasma D-Dimer level was highest in embolic infarct $(1700 \pm 964 \mathrm{ng} / \mathrm{ml})$ followed by atherothrombotic $(536 \pm 234 \mathrm{\eta g} / \mathrm{ml})$ and lowest in lacunar $(100 \pm 00 \mathrm{\eta g} / \mathrm{ml})$ subtype.
\end{abstract}

Keywords: Ischemic stroke, Plasma D-Dimer.

J Dhaka Med Coll. 2015; 24(2) : 117-125.

\section{Introduction}

The World Health Organization (WHO) has defined stroke as a clinical syndrome occurring due to sudden cerebral dysfunction producing focal rather than global neurological deficit persisting for more than 24 hours or the patient dies within 24 hours, vascular in origin, nonepileptic and non-traumatic in nature ${ }^{1}$.

Stroke is a major cause of mortality and morbidity around the world. It is the third most

1. Dr. Paritosh Kumar Sarkar, Dr. Murshed Baqui, Dr. Mohammad Shah Jahirul Hoque Chowdhury, Assistant Professor, Department of Neurology, National Institute of Neurosciences \& Hospital, Dhaka

2. Dr. Konol Saha, Registrar, Department of Neurology, Dhaka Medical College and Hospital, Dhaka

3. Dr. Md. Abdullah Alamgir, Assistant Professor, Department of Neurosurgery, National Institute of Neurosciences \& Hospital, Dhaka

4. Md. Roushan Iqbal, Registrar (In charge), Department of Surgery, BIRDEM Hospital, Dhaka

5. Dr. Swapon Kumar Ghose, Associate Professor, Department of Neurology, Dhaka Medical College \& Hospital, Dhaka

6. Dr. Mansur Habib, Professor and Head, Department of Neurology, Dhaka Medical College \& Hospital, Dhaka

7. Dr. Md Azharul Hoque, Dr. Badrul Alam Mondal, Dr. Firoz Ahmed Quraishi, Professor, Department of Neurology, National Institute of Neurosciences \& Hospital, Dhaka

8. Dr. Quazi Deen Mohammad, Director and Professor of Neurology, National Institute of Neurosciences \& Hospital, Dhaka

Correspondence : Dr. Paritosh Kumar Sarkar, Assistant Professor, Department of Neurology, National Institute of Neurosciences \& Hospital, Dhaka 
common cause of death in developed countries after coronary heart disease and cancer ${ }^{2}$. Stroke is the commonest neurological disorder of adult life and about $50 \%$ of neurological diseases among the adult admitted patients in general hospital are due to stroke. Incidence of stroke varies with age, sex, race and country to country. In general, its incidence is 150 to 300 per 100,000 populations and prevalence is 600 per 100,000 populations $^{3,4}$. It is the single most important cause of severe disability in people living in their own home. In USA the number of stroke patientsis about 700,000 per year ${ }^{5}$. Stroke caused about 4.4 millions deaths per year and one quarter of this recorded in developing countries ${ }^{6}$.

Of all stroke patients, about 85 percent are due to ischemic infarction and 15 percent are due to hemorrhage. But in a study in Bangladesh on stroke patients revealed that $57.84 \%$ are ischemic and $42.16 \%$ are hemorrhagic Stroke ${ }^{7}$.

Embolic or thrombotic occlusion of large arteries has been identified as the main cause of stroke, but other causes include abrupt occlusion of small penetrating arteries, arteritis, arterial dissection, venous occlusion and profound anemia or hyperviscosity. Unfortunately, about 35\% all ischemic events remain classified as cryptogenic ${ }^{8}$. Ischemic stroke is an etiologically heterogenous disease. Identification of the specific cause in every patient is very important because early diagnosis, acute management, assessment of clinical progression and long-term strategies is necessary to prevent recurrence ${ }^{3}$.

Haemostatic activation may be an important cause, or contributor for progression of ischemic stroke. There is evidence of increased thrombin generation and fibrin turnover, altered fibrinolytic activity, and disturbed endothelial function in acute stroke. Due to differences in the etiopathological process of different subtypes of ischemic stroke, some authors hypothesized that different stroke etiologies might be associated with a specific plasma protein expression pattern and explored the role of different proteins, such as D-Dimer as haemostatic markers in ischemic stroke s $^{3,9,10}$.

Plasma D-Dimer value is identified in patients at high risk for stroke progression and remain as an independent predictor ${ }^{11,12}$. Raised DDimer is a constant finding in thrombogenic disorders. In a study by Barber and his colleagues in 2004 reported increased levels of D-Dimer during the acute phase of ischemic stroke and related to this worse vital function and functional prognosis and to a higher risk of recurrence during follow-up ${ }^{11}$. In addition, plasma D-Dimer value is highest in association with cardioembolic mechanism. A controlled study reported that patients with cardioembolic stroke had raised mean levels of D-Dimer, and has higher risk of new embolisms ${ }^{13}$. In 2011 , Alvarez-Perez concluded in his study that there was a continuous activation of coagulation and fibrinolysis characterized by raised D-Dimer in ischemic stroke ${ }^{14}$. This study is taken for early identification of acute ischemic stroke patients in Bangladeshi population, thus stroke patients may be benefited with thrombolytic therapy with reduction of substantial morbidity and mortality.

\section{Methods}

This case control study was conducted in Dhaka Medical College Hospital (DMCH), Dhaka, from July 2010 to June 2012. Purposive sampling was done in 100 cases includes 50 cases and 50 controls within 3 days of stroke. Relevant data were collected in preformed data sheet. Age and sex matched healthy volunteers were recruited as control. Plasma D-Dimer was measured by latex agglutination method.

Patients with history of ischemic stroke within 3 days of theincidence attending in the stroke clinic of Department of Neurology or admitted in the Department of Neurology and Internal Medicine through the Outpatient and Emergency Department of DMCH were recruited for the study. The patients older than 18 years with clinical features and $\mathrm{CT}$ evidence of ischemic stroke without concomitant anticoagulation therapy were enrolled for the 
study. The patients were examined first by the investigator and finally verified by the co-guide. Clinical diagnosis of stroke was done according to National Institute of Neurological Disorders and Stroke (NINDS) criteria ${ }^{15}$. Then CT scan was done to confirm the diagnosis and volumetric measurement of the infarction done by using following formula: $A \times B \times C / 2$, where $A$ is the largest diameter and $B$ is the perpendicular diameter of the ischemic lesion, as measured, and $\mathrm{C}$ is the sum of the thickness of the slices where the lesion was visible $^{16}$. The clinical features, risk factors, other investigation findings and relevant data were collected in a preformed data sheet for each patient by the investigator. Age and sex matched healthy volunteers were recruited as control. Controls were patient's attendant, hospital staffs and voluntary blood donors. Estimation of plasma D-Dimer was done in the Department of Hematology of DMCH and was monitored by investigator and supervised by Hematologist.
Statistical analysis was performed with the help of computer by using statistical package for social science (SPSS). All data were expressed in frequency, t-test or Chi-square $\left(\chi^{2}\right)$ test or as appropriate. The levels of significance accepted at Pvalue $<0.05$.

\section{Results}

A total of 50 cases and 50 controls were included in the study and they were divided into five age groups. In ischemic stroke group, $16 \%$ patients were from 18-40 years age group and the maximum number of patients was found in $61-70$ years group which is $32 \%$. The mean $( \pm \mathrm{SD})$ of ages were $58.36 \pm 14.80$ and $58.80 \pm 15.01$ in the ischemic stroke and control group respectively. The age difference was not statistically significant $(t=0.15, p=0.990)$ between the two groups. The study comprises $34(68 \%)$ male and $16(32 \%)$ female in the ischemic stroke group while there were 36 $(72 \%)$ male and $14(28 \%)$ female in the control group. There was no significant difference in sex between two groups $\left(\chi^{2}=0.19, p=0.441\right)$ (Table-I).

Table-I

Distribution of age and sex in the study and control groups

\begin{tabular}{|c|c|c|c|c|}
\hline Age Groups (in years) & $\begin{array}{l}\text { Ischemic stroke group } \\
\qquad \mathrm{n}=50(\%)\end{array}$ & $\begin{array}{l}\text { Control group } \\
\mathrm{n}=50(\%)\end{array}$ & $\begin{array}{l}\mathrm{t} / \chi^{2} \\
\text { value }\end{array}$ & $\begin{array}{c}\mathrm{P} \\
\text { value }\end{array}$ \\
\hline $18-40$ & $8(16)$ & $8(16)$ & & \\
\hline $41-50$ & $11(22)$ & $10(20)$ & & \\
\hline $51-60$ & 7 (14) & $8(16)$ & & \\
\hline $61-70$ & $16(32)$ & $12(24)$ & & \\
\hline$>70$ & $8(16)$ & $12(24)$ & & \\
\hline Total & $50(100)$ & $50(100)$ & & \\
\hline Mean \pm SD & $58.36 \pm 14.80$ & $58.80 \pm 15.01$ & 0.15 & 0.990 \\
\hline Sex Male & $34(68 \%)$ & $36(72 \%)$ & & \\
\hline Female & $16(32 \%)$ & $14(28 \%)$ & 0.19 & 0.441 \\
\hline
\end{tabular}

Table-II

Showing Duration of symptoms and time of occurrence of stroke

\begin{tabular}{lc}
\hline Variables & Ischemic stroke group $\mathrm{n}=50(\%)$ \\
\hline Duration of symptoms (hours) & $33.49 \pm 28.71$ \\
Time of occurrence & \\
Sleep & $19(38)$ \\
Work & $31(62)$ \\
\hline
\end{tabular}


The occupational status of the study subjects were placed in five categories. The categories were service holders, businessmen, laborers, farmers and house wives. In the control group $36 \%, 30 \%, 10 \%$, and $24 \%$ were service holders, businessmen, laborers and house wives while in the case group $10 \%, 8 \%, 22 \%, 28 \%$ and $32 \%$ were service holders, businessmen, laborers, farmers and house wives respectively. The occupational status between the two groups differ significantly $\left(\chi^{2}=30.53, p=0.001\right)$ (Fig. -1$)$. Regarding the economic status, $62 \%, 34 \%$ and $4 \%$ were in lower, middle, higher economic class in ischemic stroke group; while 34\%, 40\% and $26 \%$ in lower, middle and higher economic class in control group respectively. There was significant statistical difference between the two groups regarding the economic class $\left(\chi^{2}=12.39, p=0.002\right)$ (Fig.-2).

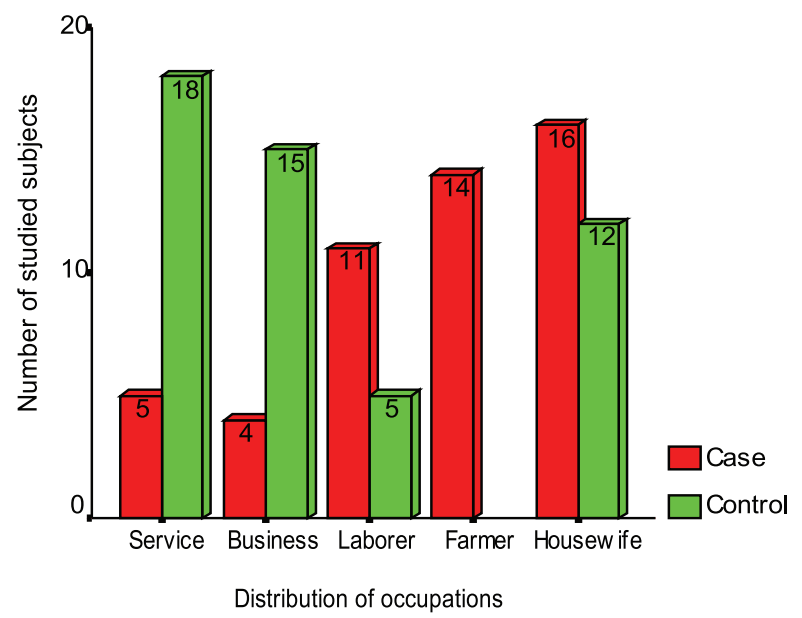

Fig.-1: Distribution of occupations in the studied subjects

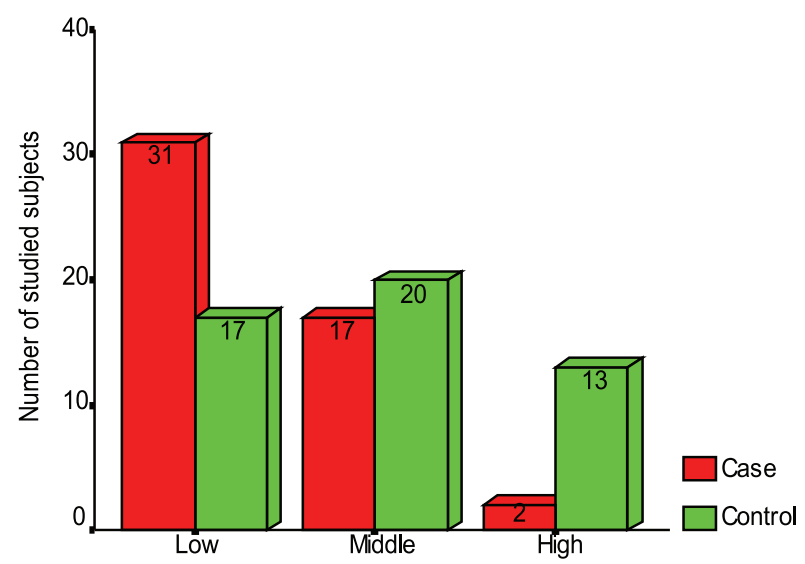

Distribution of economical class in the sutdied subjects

Fig.-2: Distribution of economical status in the studied subjects
In ischemic stroke group all patients presented within 4 hours to 3 days. The mean duration of symptoms was $33.49 \pm 28.71$ hours. The study showed that $62 \%$ patients had the attack during work and remaining 38\% had attack at sleep (Table-II). Majority of patients presented with hemiplegia (98\%) and speech disturbances (86\%). Only few patients presented with unconsciousness $(12 \%)$ and seizure $(8 \%)$ (Table-III).

Table-III

Clinical presentation in cases

\begin{tabular}{lc}
\hline Variables & $\begin{array}{c}\text { Ischemic stroke group } \\
\mathrm{n}=50(\%)\end{array}$ \\
\hline Hemiplegia & $49(98)$ \\
Speech involvement & $43(86)$ \\
Unconsciousness & $6(12)$ \\
Seizure & $4(8)$ \\
\hline
\end{tabular}

Hypertension is the most prevalent risk factor found in $58 \%$ patients. Only $3(6 \%)$ patients had history of diabetes and $5(10 \%)$ patients had valvular heart disease. About $62 \%$ patients had habit of smoking (Table-IV).

Table-IV

Risk factors in the studied subjects

\begin{tabular}{lc}
\hline Variables & $\begin{array}{c}\text { Ischemic stroke group } \\
\mathrm{n}=50(\%)\end{array}$ \\
\hline Hypertension & $29(58)$ \\
Diabetes & $3(6)$ \\
Valvular heart disease & $5(10)$ \\
Family H/O Stroke & $15(30)$ \\
Smoking & $31(62)$ \\
\hline
\end{tabular}

Glasgow Coma Scale (GCS) score was observed in all patients. A score of 9-15 was found in $88 \%$ patients and a score $<8$ was found in $12 \%$ patients. A variety of speech involvement found in the studied group. Dysarthria and motor dysphasia occurred in 40\% patients each. Global dysphasia was found only in $6 \%$ patients. However, speech was normal in $14 \%$ patients. Cranial nerve lesion was found in $82 \%$ patients (Table-V). 
Table-V

Neurological findings in the studied subjects

\begin{tabular}{lc}
\hline Variables & $\begin{array}{c}\text { Ischemic stroke group } \\
\mathrm{n}=50(\%)\end{array}$ \\
\hline GCS Score & $44(88)$ \\
Score 9-15 & $6(12)$ \\
Score > 8 & \\
Speech involvement & $7(14)$ \\
Normal & $20(40)$ \\
Motor dysphasia & $3(6)$ \\
Global dysphasia & $20(40)$ \\
Dysarthria & $41(82)$ \\
Cranial nerve involvement &
\end{tabular}

Hematological and biochemical findings of the studied subjects are shown in Table-6. The mean values of serum cholesterol, triglyceride, LDL and HDL were $219 \pm 50,181 \pm 70,133 \pm 32$ and $41 \pm 9$ respectively (Table-VI).

Table-VI

Hematological findings in the studied subjects

\begin{tabular}{lc}
\hline Variables & $\begin{array}{c}\text { Ischemic stroke group } \\
\mathrm{n}=50\end{array}$ \\
\hline Hematological values & \\
Total count of WBC & $9243 \pm 2478$ \\
Polymorph (\%) & $70 \pm 9$ \\
RBC (million/ $\mathrm{mm}^{3}$ ) & $4.4 \pm 0.64$ \\
Platelet count (per $\mathrm{mm}^{3}$ ) & $2,46,780 \pm 56,000$ \\
Hemoglobin (gm/dl) & $12.6 \pm 1.4$ \\
ESR mm in $1^{\text {st }}$ hour & $32 \pm 18$ \\
Plasma glucose (mmol/L) & $7.9 \pm 3.6$ \\
Serum creatinine (mg/dl) & $1.07 \pm 0.18$ \\
Lipid profile & \\
Serum triglyceride (mg/dl) & $181 \pm 70$ \\
Serum LDL (mg/dl) & $133 \pm 32$ \\
Serum cholesterol (mg/dl) & $219 \pm 50$ \\
Serum HDL (mg/dl) & $41 \pm 9$ \\
\hline
\end{tabular}

Values are expressed in mean $\pm \mathrm{SD}$

CT scan of brain was performed in all patients; and the site and volume of the lesion was measured. The study shows, both cortical and subcortical lesion, were found in $56 \%$ patients, whereas subcortical lesion was found in 34\% patients and cortical lesion in 10\% patients only. CT volume (mean \pm SD) of the involved brain lesion was $19.95 \pm 4.92 \mathrm{~cm}^{3}$ (Table-VII).

Table-VII

Findings of CT scan of the brain in the studied subjects

\begin{tabular}{lc}
\hline Variables & $\begin{array}{c}\text { Ischemic stroke group } \\
\mathrm{n}=50(\%)\end{array}$ \\
\hline Site of the lesion & $5(10)$ \\
Cortical & $28(56)$ \\
Cortical and subcortical & $17(34)$ \\
Subcortical & $19.95 \pm 4.92$ \\
\hline
\end{tabular}

Qualitative and quantitative measurement of plasma D-Dimer was made in both studied subjects and control groups. Qualitative analysis of plasma D-Dimer reveals $76 \%$ positive and $24 \%$ negative in the ischemic stroke group. On the other hand, plasma DDimer value was only $6 \%$ positive and $94 \%$ negative in the control group. Qualitative analysis of plasma D-Dimer differs significantly $\left(\chi^{2}=50.64, p=0.001\right)$ between the two groups. The analysis also reveals $76 \%$ sensitivity and $94 \%$ specificity of the Plasma D-Dimer test. Quantitative measurement of plasma D-Dimer ( $\mathrm{p}=0.001)$ was $804 \pm 120 \mathrm{ng} /$ $\mathrm{ml}$ and $126 \pm 16 \mathrm{ng} / \mathrm{ml}$ in ischemic and control groups respectively. The plasma concentration of D-Dimer between the two groups was statistically highly significant $(\mathrm{t}=31.21$, $\mathrm{p}=0.001)$ (Table-VIII).

There were 24\% lacunar infarcts, 40\% atherothrombotic and 36\% embolic infarcts in the studied group. Mean plasma D-Dimer level was highest in embolic infarct $(1700 \pm 964 \mathrm{\eta g} /$ $\mathrm{ml})$ followed by atherothrombotic $(536 \pm 234 \mathrm{\eta g} /$ $\mathrm{ml})$ and lowest in lacunar $(100 \pm 00 \eta \mathrm{g} / \mathrm{ml})$ subtype. A significant difference $(\mathrm{F}=31.16$, $\mathrm{p}=0.001$ ) observed in the mean plasma $\mathrm{D}$-Dimer level in the subtypes of stroke (Table-IX). 
Table-VIII

Qualitative and quantitative analysis of Plasma D-Dimer

\begin{tabular}{lcccccc}
\hline Variables & $\begin{array}{c}\text { Ischemic } \\
\text { stroke group } \\
\mathrm{n}=50(\%)\end{array}$ & $\begin{array}{c}\text { Control group } \\
\mathrm{n}=50(\%)\end{array}$ & Sensitivity & Specificity & $\chi^{2} / \mathrm{t}$ & $\mathrm{p}$ value \\
D-Dimer & $38(76)$ & $3(6)$ & $76 \%$ & $94 \%$ & 50.64 & 0.001 \\
\hline Positive & $12(24)$ & $47(94)$ & & & & \\
Negative & $50(100)$ & $50(100)$ & & & & \\
Total & $804 \pm 120$ & $126 \pm 16$ & & & 31.21 & 0.001 \\
Quantitative assay $(\eta \mathrm{ng} / \mathrm{ml})$ & & & & \\
\hline
\end{tabular}

Table-IX

Mean value of Plasma D-Dimer in etiological subtypes of acute ischemic stroke

\begin{tabular}{lcccc}
\hline $\begin{array}{l}\text { Subtypes of Acute } \\
\text { ischemic stroke }\end{array}$ & $\begin{array}{c}\text { Number of patients } \\
\mathrm{n}=50(\%)\end{array}$ & $\begin{array}{c}\text { Plasma D-Dimer } \\
(\eta \mathrm{g} / \mathrm{ml})\end{array}$ & $\mathrm{F}$ & $\mathrm{p}$ value \\
\hline Lacunar & $12(24)$ & $100 \pm 00$ & & \\
Atherothrombotic & $20(40)$ & $536 \pm 234$ & 31.16 & 0.001 \\
Embolic & $18(36)$ & $1700 \pm 964$ & & \\
\hline
\end{tabular}

\section{Discussion}

Rapid diagnosis of stroke remains a major challenge for patient management and therapeutic interventions. Diagnosis of acute ischemic stroke is difficult because CT scan results may appear normal in the early stage or in patients with minor symptoms, and MRI is not always possible in the early hours of the disease. Therefore, the first objective in the management with patients of ischemic stroke is the diagnosis of subtypes as thrombolytic agents have been shown to improve outcome when therapeutic intervention is taken timely. Objective of the present study was to assess the potential diagnostic utility of blood borne protein biomarker in predicting stroke. Plasma D-Dimer level was measured in 50 acute ischemic stroke patients and was compared with the age and sex matched healthy control groups. The results of the present study demonstrate that D-Dimer level significantly differ among the stroke subtypes after an acute ischemic event and the measurement of DDimer levels can be reliable in the early diagnosis of the mechanism of underlying cause. The mean age of first stroke in male is 68.6 years and 72.9 years in female; and male incidence of stroke rate is 33\% higher in comparison to female in Western population ${ }^{17}$ but in a series of 291 consecutive stroke patients in Bangladesh revealed mean age was 57.9 years and incidence of stroke was $80 \%$ in male patients ${ }^{18}$. This study reveals mean age of stroke as $58.36 \pm 14.80$ years and frequency of stroke in male and female patients are 68\% and $32 \%$ respectively. A study from Chittagong Medical College, Bangladesh demonstrates that middle class group has the highest risk of ischemic strokes and housewives are most commonly affected group ${ }^{19}$. Our study also demonstrates higher incidence of stroke in middle socioeconomic class (34\%) and the commonest in housewives occupation (32\%). Habit of smoking (62\%) was higher in this study comparison to Hossain's study from Bangladesh $^{20}$.

Hemostatic abnormalities after cerebral ischemia apparently are not related to the extent of the neurological damage in terms of severity and duration of symptoms but to the mechanism responsible for cerebral ischemia. ${ }^{21}$ In our study analysis of symptoms reveal that hemiplegia (98\%), speech involvement (86\%), cranial nerve involvement $(82 \%)$ correlate with the findings of Walter his colleagues. ${ }^{21}$ In a study of Aydin et al., found $74 \%$ of patients with GCS $>13$ and $11 \%$ with 
GCS $<7^{22}$. In our study $88 \%$ patients were with GCS score $9-15$ and $12 \%$ were with score $<8$. In a study in Australia by Kawn\& Hand in 2006, did not find significant correlation on clinical characteristics of stroke on admission with blood glucose, serum creatinine, and platelet counts, but had significant association with hemoglobin and leukocyte count ${ }^{23}$. This study has similar relationship with the above parameters except blood glucose level and platelet count and also significant association with other variables, as for example, lipid profile, ESR, total and differential count of leukocytes.

At present non-contrast CT scan is the standard of care in the evaluation of acute stroke, although CT changes associated with ischemia are often absent or quite subtle ${ }^{24}$. In this study patients were categorized into stroke subtypes on the basis of clinical history, examination and CT findings. Cerebral infarction was categorized anatomically into 3 groups: cortical, subcortical and both cortical and subcortical; and our study reveals both cortical and subcortical 56\%; subcortical 34\% and cortical only $10 \%$ cases which is similar to several studies $25,26$.

Several studies have found that an increased concentration of plasma D-Dimer level in acute ischemic stroke patients ${ }^{27,28,29}$. This D-Dimer value showed weak to moderate correlation, supports a link between inflammation, hypercoagubility and fibrin turnover in ischemic stroke patients ${ }^{27}$. On the other hand, Alvarez-Perez and Montaner, in their separate study found significant correlation of plasma D-Dimer with a mean value ranging from 500$218 \mathrm{ng} / \mathrm{ml}$ in different subtypes with the lowest concentration in atherothrombotic group ${ }^{14,25}$. Park et al., found mean plasma D-Dimer level on admission as $215.3 \mathrm{\eta g} / \mathrm{ml}$ in patients with focal infarction and $385.7 \mathrm{ng} / \mathrm{ml}$ in patients with multiple embolic infarctions ${ }^{16}$. He also observed that plasma D-Dimer value was 566 $\eta \mathrm{g} / \mathrm{ml}$ in patients with $1-19 \mathrm{cc}$ infarctions, 668 $\eta \mathrm{g} / \mathrm{ml}$ in patients with $20-49 \mathrm{cc}$ infarctions, 702 $\eta \mathrm{g} / \mathrm{ml}$ in 50-199 cc infarctions, $844 \mathrm{\eta g} / \mathrm{ml}$ in
$>200$ cc infarctions ${ }^{16}$. These findings are also similar with a study from a medical university from Japan ${ }^{30}$. Although the volumetric measurement of CT scan of the brain and Plasma D-Dimer value correlated on admission but the D-Dimer value was insignificant with the stroke volume at 7 days after stroke ${ }^{16}$. In this study the average CT volume of lesion was $19.95 \pm 4.92 \mathrm{~cm}^{3}$ and Plasma D-Dimer measured within $33.49 \pm 28.71$ hours after the event of stroke. Our study reveals that mean concentration of plasma D-Dimer was $804 \pm 120$ $\eta \mathrm{g} / \mathrm{ml}$ in all ischemic stroke patients irrespective of subtypes and a value $126 \pm 16 \eta$ $\mathrm{g} / \mathrm{ml}$ in control group. Furthermore, the plasma D-Dimer $(\eta \mathrm{g} / \mathrm{ml})$ concentration was $1700 \pm 964$, $536 \pm 234$ and $100 \pm 0$ in embolic, atherothrombotic and lacunar group respectively. Values of plasma D-Dimer varied significantly in different subtypes of stroke in this study although we found lowest concentration in lacunar group which is contrary to the other studies $^{14,} 25$ but similar to Ageno's study ${ }^{21}$. Some authors have shown that plasma DDimer has a specificity of $68 \%$ to $96 \%$ and a sensitivity of $61 \%$ to $72 \%$ when tested by ELISA $\operatorname{method}^{10,21}$. This study reveals that plasma D-Dimer has a specificity of $94 \%$ and a sensitivity of $76 \%$ in ischemic stroke patients.

This study suggests that plasma D-Dimer level is a potential biomarker in acute ischemic stroke and also helpful in categorizing the subtypes. Estimation of plasma D-Dimer level and adjunct to $\mathrm{CT}$ scan will strengthen the diagnosis of acute ischemic stroke which will help in therapeutic management.

\section{Conclusion}

This study indicates that raised plasma DDimer is a constant finding in acute phase of ischemic stroke. Plasma D-Dimer has statistical significance with neurological involvement and also with the changes of $\mathrm{CT}$ scan diameter or thickness of lesion. Its sensitivity and specificity are higher in ischemic stroke group. Measurement of plasma D-Dimer is simple and can be an adjunct to diagnosis of acute ischemic stroke. 
Thus measuring plasma D-Dimer level will further strengthen the clinical diagnosis of ischemic stroke particularly where CT scan could not be done.

\section{References}

1. Aho K, Harmsen P, Hartano S, et al. Cerebro vascular disease in the community, Results of WHO collaboration study. World Health Organ Bull. 1980; 58:113-30.

2. Poungvarin N. Stroke in developing world. Lancet. 1998; 352 (Supp1-III):19-22.

3. Sudlow CLM, Warlow CP. Comparable studies of the incidence of stroke and its pathological types: Results from and international collaboration. Stroke. 1997; 28: 491-9.

4. Mohammad QD, Habib M, Hoque MA, Mondol MBA, Haque B, Hossain S, et al. Prevalence of stroke in a Bangladeshi population aged forty years and above. Mymensingh Med J. 2011; 20(4): 640-4.

5. Lloyd-Jones D, Adams R. Stroke. In: Heart disease and stroke statistics-2009 update: a report from the American Heart Association. Statistics Committee and Stroke Statistics Subcommittee. Circulation. 2009; 119(3):480-6.

6. Murray CJL, Lopez AD. Mortality by cause for eight regions of the world: global burden of disease study. Lancet. 1997; 349(9061):1269-76.

7. Alam B, Habib M, Quraishi FA, Haque B, Hoque A, Mohammad QD. Stroke evaluation of risk factors. Bangladesh J Neurosci 1999; 15(2):14-8.

8. Whiteley W, Chong WL, Sengupta A, Sandercock P. Blood markers for the prognosis of ischemic stroke: a systematic review. Stroke. 2009; 40(5): e380-9.

9. Saenger AK, Christenson RH. Stroke biomarkers: Progress and challenges for diagnosis, prognosis, differentiation and treatment. Clinical Chemistry. 2010; 56(1):21-33.

10. Whiteley W, Tseng MC, Sandercock P. Blood biomarkers in the diagnosis of ischemic stroke: a systematic review. Stroke. 2008; 39: 2902-9.

11. Barber M, Langhorne P, Rumley A, Lowe DG, Stott DJ. Haemostatic Function and progressing ischemic stroke: D-Dimer predicts early clinical progression. Stroke. 2004; 35:1421-30.

12. Barber M, Langhorne P, Rumley A, Lowe DG, Stott DJ. D-Dimer Predicts Early clinical progression in ischemic stroke: Confirmation using routine clinical assays. Stroke. 2006; 37:1113-5.

13. Lip GYH, Lowe GDO, Rumley A et al. Increased marker of thrombogenesis in chronic atrial fibrillation: effects of warferin treatment. Br Heart J. $1995 ; 73: 527-33$.

14. Alvarez-Perez FG, Castelo-Branco M, AlvarezSabin. Usefulness of measurement of fibrinogen, D-Dimer, D-dimer/fibrinogen ratio, C-reactive protein and erythrocytes sedimentation rate to assess the pathophysiology and mechanism of ischemic stroke. J NeurolNeursurgPsychiatr. 2011.

15. Kidwell SC, Warach S. Acute ischemic cerebrovascular syndrome: diagnostic criteria. Stroke. 2003; 34: 2995-8.

16. Park YW, Koh EJ and Choi HY. Correlation between serum D-Dimer level and volume in Acute ischemic stroke. J Korean Neurosurg Soc. 2011; 50: $89-94$

17. Appelros P Stegmayr B, Terent A. Sex Differences in Stroke Epidemiology: A Systematic Review. Stroke. 2009; 40: 1082-90.

18. Chowdhury AH, Yokoyama T, Kokubo Y, Zaman MM, Haque A, Tanaka H. Apolipoprotein E genetic polymorphism and stroke subtypes in a Bangladeshi hospital-based study. J Epidemiol. 2001; 11: 131-8.

19. Siddique MAN, Nur Z, Mahabub MS, Alam MB, Miah MT. Clinical presentation and epidemiology of stroke - a study of 100 cases. J Medicine. 2009; 10: 86-9.

20. Hossain MZ, Ahmed SU, Sarder MH, Dasgupta R, Das A, Sarker RN, et al. Analysis of risk factors associated with stroke in young Adults: A prospective study. J Dhaka Med Coll. 2009; 18(2): 95-100.

21. Ageno W, Finazzi S, Steidl L, Biotti MG, Mera V, Melzid'Eril GV, et al. Plasma measurement of DDimer levels for the early diagnosis of ischemic stroke subtypes. Arch Intern Med. 2002; 162:258993.

22. Aydin SA, Koksal O, Bulut M, Ozucelik DN, Ozdemir F. Clinical value of D-dimer and other coagulation markers in differential diagnosis of hemorrhagic and ischemic stroke. AkademikAcil Tip Dergisi. 2009; 8:38-42.

23. Kwan J, Hand P. Early neurological deterioration in acute stroke: Clinical characteristics and impact on outcome. Q J Med. 2006; 99:625-33.

24. Laskowitz DT, Kasner SE, Saver J, Remmel KS, Jauch EC and Brain Study Group. Clinical usefulness of a biomarker-based diagnostic test for acute stroke: The biomarker rapid assessment in ischemic injury (Brain) study. Stroke. 2009; 40:77-85. 
25. Montaner J, Perea-Gainza M, Delgado P, Ribo M, Chacon $\mathrm{P}$, Rosell A, et al. Etiologic diagnosis of ischemic stroke subtypes with plasma biomarkers. Stroke. 2008; 39(8):2280-7.

26. Bamford J, Sandercock P, Dennis M, Burn J, Warlow C. Classification and natural history of clinical subtypes of cerebral infarction. Lancet. 1991; 337: 1521-6.

27. Napoli MD, Papa F. Inflammation, Hemostatic Markers, and Antithrombotic Agents in Relation to Long-Term Risk of New Cardiovascular Events in First-Ever Ischemic Stroke. Stroke. 2002; 33: 1763-71.

28. Hankay G, Warlow C. Treatment and secondary prevention of stroke: evidence, costs, and effects on individuals and populations. Lancet. 1999; 354: 1457-63.

29. Sacco RL, Wolf PA, Kannel WB, McNamara PM. Survival and recurrence following stroke: the Framingham study. Stroke. 1982; 13: 290-6.

30. Tohgi H, Kawashima M, Tamura K, Suzuki H. Coagulation-fibrinolysis abnormaltities in acute and chronic phases of cerebral thrombosis and embolism. Stroke. 1990; 21: 1663-7. 